\title{
Identification of PAQR3 as a new candidate tumor suppressor in hepatocellular carcinoma
}

\author{
HONG GE WU ${ }^{1}$, WEN JIE ZHANG ${ }^{3}$, QIAN DING ${ }^{1}$, GANG PENG $^{1}$, ZHEN WEI ZOU ${ }^{1}$, \\ TING LIU ${ }^{2}$, RU BO CAO ${ }^{1}$, SHI JIANG FEI ${ }^{1}$, PENG CHENG LI ${ }^{1}$, KUN YU YANG ${ }^{1}$, \\ JIAN LI HU ${ }^{1}$, XIANG FANG DAI ${ }^{1}$, GANG WU ${ }^{1}$ and PIN DONG LI ${ }^{1}$ \\ ${ }^{1}$ Cancer Center and ${ }^{2}$ Department of Infectious Diseases, Union Hospital, Tongji Medical College, \\ Huazhong University of Science and Technology, Wuhan, Hubei $430023 ;{ }^{3}$ Department of Pathology, \\ Shihezi University School of Medicine, Shihezi, Xinjiang 832002, P.R. China
}

Received July 16, 2014; Accepted September 2, 2014

DOI: $10.3892 /$ or.2014.3532

\begin{abstract}
Progestin and adipoQ receptor family member III (PAQR3) is a regulator that negatively modulates the Ras/Raf/ MEK/ERK signaling cascade and the GPCR G $\beta \gamma$ subunit signaling pathway. The role of PAQR3 in hepatocellular carcinoma (HCC) has not been elucidated. The present study investigated the expression of PAQR 3 and its prognostic value in primary HCC patients. Furthermore, the functional aspects of PAQR3 were also studied using an in vitro cell model. PAQR3 expression was examined in paired HCC and adjacent noncancerous tissues using real-time quantitative RT-PCR (62 pairs) and western blotting (26 pairs). We also analyzed PAQR3 expression in 132 additional HCC samples by immunohistochemistry. The functional impact of PAQR3 on the proliferation and colony formation of an HCC cell line was analyzed by transfecting cells with a full-length PAQR3 expression vector or siRNA targeting PAQR3. The expression of PAQR3 was significantly decreased in the cancer tissues. Clinicopathological analyses showed that the expression of PAQR 3 was significantly correlated with expression of serum $\alpha$-fetoprotein (AFP), mitotic count, tumor size, histological grade and recurrence. Notably, Kaplan-Meier survival curves revealed a correlation between decreased expression of PAQR3 and the poor prognosis of HCC patients. Multivariate analyses showed that PAQR3 expression is an independent prognostic marker for overall and disease-free survival of HCC patients. Furthermore, restoring PAQR3 expression in HCC cells
\end{abstract}

Correspondence to: Dr Gang Wu or Dr Pin Dong Li, Cancer Center, Union Hospital, Tongji Medical College, Huazhong University of Science and Technology, 156 Xinghua Road West, Wuhan, Hubei 430023, P.R. China

E-mail: minichen_41@163.com

E-mail: 1pd8204@163.com

Key words: PAQR3, expression, hepatocellular carcinoma, prognosis, tumor suppressor significantly diminished Hep3B cell proliferation and colony formation. Silencing PAQR3 expression in hepatic normal cell line LO2 significantly enhanced cell growth. PAQR3 may play an important role in the progression of $\mathrm{HCC}$ and serve as a potential candidate for the targeted therapy of HCC.

\section{Introduction}

Hepatocellular carcinoma (HCC) is one of the most deadly and prevalent malignant diseases, not only in developing countries but also in the US and Europe, and is therefore a serious disease burden for public health (1). In recent years, the morbidity and mortality rates of HCC have been continuously rising, placing HCC as the second leading cause of deaths among nine most common cancers in China (2). Due to the lack of sensitive and specific biomarkers in the clinic, the existing early-stage screening strategies have limited benefits to the prevention of HCC. Even with advanced treatments, such as surgery, chemotherapy and radiotherapy, HCC still presents a dismal prognosis with an overall survival rate as low as $25-39 \%$ (3). Therefore, there is an urgent need to identify new tumor markers and novel targeted agents for the early diagnosis of $\mathrm{HCC}$ and develop efficacious regimens for treating $\mathrm{HCC}$ in order to improve patient outcome.

In the past few decades, many cellular signaling pathways and genes have been implicated in the pathogenesis of HCC, including the Ras/Raf/MEK/ERK signaling pathway (4-7). Progestin and adipoQ receptor family member III(PAQR3), also named RKTG, negatively regulates the Ras/Raf/MEK/ERK signaling cascade and the $\mathrm{G}$ protein-coupled receptor (GPCR) G $\beta \gamma$ subunit signaling pathway $(8,9)$. PAQR3 is a member of the PAQR family of proteins comprising an intriguing group of recently discovered receptors and a seven transmembrane protein localized at the Golgi apparatus $(8,10)$. PAQR3 expression inhibits EGF-stimulated ERK and RSK phosphorylation, blocks NGF-mediated cell differentiation, and antagonizes Ras and Raf-1-stimulated Elk-1 transactivation (8). Through interaction with Raf-1, PAQR3 alters the localization of Raf-1 from the cytoplasm to the Golgi apparatus, blocks EGF-stimulated Raf-1 membrane translocation, and reduces the interaction of Raf-1 with Ras and MEK1 (11). PAQR3 has a negative effect 
on cell proliferation, migration, sprouting and angiogenesis of endothelial cells by suppressing mitogen-activated protein kinase signaling (12). In addition, PAQR3 negatively regulates the transactivation activity of hypoxia-inducible factor $1 \alpha$ (HIF-1 $\alpha$ ) by inhibiting formation of the HIF- $1 \alpha / \mathrm{p} 300$ complex and suppressing VEGF transcription, thereby reducing hypoxia-induced VEGF production (12). Furthermore, recent studies have found that PAQR3 is implicated in a set of human cancers, including clear-cell renal cell carcinoma and colorectal cancer $(12,13)$. However, the significance of altered expression of PAQR3 in HCC has not been fully elucidated due to the lack of studies using fresh human tumor samples.

In the present study, we investigated the expression of PAQR3 in primary HCC using real-time quantitative RT-PCR, western blotting and immunohistochemistry. We also identified the relationship between PAQR3 expression and clinicopathological features of the HCC cases and evaluated the potential prognostic value of PAQR3 expression for the survival of HCC patients. In addition, we proved that PAQR3 can inhibit tumor cell growth and colony formation in vitro. To the best of our knowledge, the data generated in the present study represent the first report correlating the presence of PAQR3 with clinicopathological characteristics and the survival of HCC patients.

\section{Materials and methods}

Ethics statement. This study was approved by the Institutional Ethics Review Board (IERB) of Union Hospital, and written informed consent was obtained from all patients studied.

Cell lines and culture conditions. The hepatic cancer cell lines, Hep-3B, Hep-G2 and Huh-7, and the normal liver cell line LO2 were obtained from the Committee of Type Culture Collection of the Chinese Academy of Sciences (Shanghai, China). Cell lines were cultured in RPMI-1640 media supplemented with $10 \%$ heat-inactive fetal bovine serum (FBS). The cells were incubated at $37^{\circ} \mathrm{C}$ in a humidified chamber containing $5 \% \mathrm{CO}_{2}$.

Patients and tissue samples. To investigate $\mathrm{PAQR} 3$ expression in HCC, 62 HCC tissues and paired adjacent non-cancerous tissues, not less than $2 \mathrm{~cm}$ away from the HCC tissues, were collected respectively from the HCC patients undergoing hepatectomy at Union Hospital between March and June 2012. The diagnosis of HCC was confirmed by histopathology. Surgically removed fresh tissues were immediately frozen in liquid nitrogen and stored at $-70^{\circ} \mathrm{C}$ until use. In addition, another 132 paraffin-embedded $\mathrm{HCC}$ tissues were collected between 2004 and 2007. These patients did not receive preoperative chemotherapy or radiotherapy.

The follow-up data of the HCC patients included in this study were obtained as a routine practice. Our outpatient department performed postoperative follow-up on the HCC patients at intervals of every 3 months for the first 2 years, every 6 months from the 3rd to the 5th year, and annually thereafter for an additional 5 years, or until patient death, whichever occurred first. Overall survival was defined as the time from surgery to patient death or the last follow-up and was used as a measure of prognosis, while disease-free survival was defined as the time from surgery to disease recurrence or metastasis.
The histological types of HCC were defined according to the classification criteria of WHO.

RNA extract preparation and quantitative real-time $P C R(q R T-P C R)$. Total RNA extracts were prepared using TRIzol reagent (Invitrogen; 15596018) according to the manufacturer's protocol. Next, RNA $(2 \mu \mathrm{g})$ was reversely transcribed into first strand cDNA by M-MLV reverse transcriptase (Promega; M1705) according to the manufacturer's instructions. PAQR3 and GAPDH were then amplified by quantitative real-time PCR using the following primers: PAQR3 forward, 5'-ATGGCAAAGGCTCCGTTCT-3' and reverse, 5'-AGTTTAGTTGTCCTGGAAAGTACCG-3'; GAPDH forward, 5'-CTCCTCCTGTTCGACAGTCAGC-3' and reverse, 5'-CCCAATACGACCAAATCCGTT-3'. Gene specific amplification was performed in an ABI 7900HT real-time PCR system (Life Technologies, USA) with a $15-\mu \mathrm{l}$ PCR mix containing $0.5 \mu \mathrm{l}$ of cDNA, $7.5 \mu \mathrm{l}$ of $2 \mathrm{X}$ SYBR-Green Master Mix (Invitrogen; 11760500) and $200 \mathrm{nM}$ of the appropriate primers. The mixture was preheated for $10 \mathrm{~min}$ at $95^{\circ} \mathrm{C}$ followed by 45 cycles of amplification (30 sec at $95^{\circ} \mathrm{C}$ and $1 \mathrm{~min}$ at $60^{\circ} \mathrm{C}$, respectively). The resolution curve was measured at $95^{\circ} \mathrm{C}$ for $15 \mathrm{sec}, 60^{\circ} \mathrm{C}$ for $15 \mathrm{sec}$ and $95^{\circ} \mathrm{C}$ for $15 \mathrm{sec}$. The $\mathrm{Ct}$ (threshold cycle) value of each sample was calculated, and the relative expression of PAQR3 mRNA was normalized to the GAPDH value (2-ACt method).

Preparation of total protein extracts and western blot analysis. The hepatic cancer cells and frozen HCC samples including tumor or non-tumor tissue control were homogenized in RIPA lysis buffer. After centrifugation at $12,000 \mathrm{rpm}$, at $4^{\circ} \mathrm{C}$ for $20 \mathrm{~min}, \sim 25 \mu \mathrm{g}$ of the protein samples was run on a $12 \%$ SDS-PAGE gel and transferred to polyvinylidene difluoride membranes (PVDF, Millipore). After blocking non-specific binding sites for 45 min with 5\% non-fat milk, the membranes were incubated with goat polyclonal antibody against PAQR3 (1:300) and GAPDH (1:1,000; both from Santa Cruz, CA, USA) at $4^{\circ} \mathrm{C}$ overnight, respectively. The membranes were then washed with TBST for three times, (15 min each time) and then incubated with HRP-conjugated anti-goat secondary antibody $(1: 10,000)$ for $45 \mathrm{~min}$ at room temperature. The membranes were developed by an enhanced chemiluminescence system (ECL; Cell Signaling) after being washed with TBST for three times. The intensity of the protein bands was determined by densitometry using ImageJ software (Image J; National Institutes of Health).

Immunohistochemistry and PAQR3 immunostaining scoring principle. Formalin-fixed and paraffin-embedded tissues were sectioned $(4-\mu \mathrm{m})$ for immunohistochemical analysis. The sections were deparaffinized and rehydrated through a graded series of aqueous ethanol solutions. For the antigen retrieval, the slides were immersed in EDTA [1 mmol/l, $(\mathrm{pH} \mathrm{8.0)]} \mathrm{and}$ boiled for $20 \mathrm{~min}$ in a microwave oven. After rinsing with PBS, endogenous peroxidase was blocked with $0.3 \%$ hydrogen peroxide for $15 \mathrm{~min}$ at room temperature. The slides were incubated with the primary antibody (1:100; Santa Cruz), in a humidified chamber at $4^{\circ} \mathrm{C}$ overnight. Following additional washing with PBS for three times, the sections were sequentially incubated with horseradish peroxidase-conjugated 
secondary antibody (Envision ${ }^{\mathrm{TM}}$ detection kit; GK500705; Gene Tech) at $37^{\circ} \mathrm{C}$ for $30 \mathrm{~min}$, and then washed three times with PBS. Finally, 3,3'-diaminobenzidine tetrahydrochloride (DAB) was used for the signal development and then the sections were lightly counterstained with $20 \%$ hematoxylin. The slides were dehydrated and mounted on coverslips. For the negative controls, PBS was used in place of the primary antibody.

The total PAQR3 immunostaining score was calculated as the sum of the percent positivity of the stained tumor cells and the staining intensity. The percent positivity was scored as ' 0 ' ( $<5 \%$, negative), ' 1 ' (5-25\%, sporadic), ' 2 ' $(>25-50 \%$, focal), ' 3 ' ( $>50 \%$, diffuse). The staining intensity was scored as ' 0 ' (no staining), ' 1 ' (weakly stained), '2' (moderately stained), and ' 3 ' (strongly stained). Both the percent positivity of cells and the staining intensity were determined under double-blind conditions. The PAQR3 immunostaining score was expressed as the value of the percent positivity score multiply the staining intensity score, which ranged from 0 to 9 . The PAQR3 expression levels were defined as follows: - (score 0-1), + (score 2-3), ++ (score 4-6) and +++ (score >6). Based on the PAQR3 expression levels, the HCC patients were divided into two groups: low PAQR3 expression group (PAQR3- or PAQR3+) and high PAQR3 expression group (PAQR3++ or PAQR3+++).

Expression plasmid and transient transfections. A eukaryotic expression plasmid pcDNA3.1(+) containing full-length human PAQR3 cDNA was obtained from the GenePharma Company (Shanghai, China). Empty vector was used as the negative control. Hep-3B cells were cultured in 6-well plates until they reached $85-90 \%$ confluency, and then transient transfections were performed using Lipofectamine 2000 (Invitrogen) according to the manufacturer's instructions. Forty-eight hours after transfection, gene expression was examined by western blot analysis, and then cell proliferation and colony formation analyses were performed.

RNA oligonucleotides and cell transfections. To silence PAQR3 expression, siRNAs were synthesized by GenePharma Company (Shanghai, China). The siRNA sequences were as follows: siRNA-PAQR3 sense, 5'-CGCAGAUUCAUCCCAA UUATT-3' and antisense, 5'-AUAUGCCAUAUUUGGUGG CTT-3'; the negative control (NC) sense, 5'-UUCUCCGAACG UGUCACGUTT-3' and antisense, 5'-ACGUGACACGUUCG GAGAATT-3'. Four hundred picomoles siRNA-PAQR3 or NC was transfected into $2 \times 10^{5} \mathrm{LO} 2$ cells using Lipofectamine RNAi MAX reagent (Invitrogen, USA) according to the manufacturer's protocol. After that, cell proliferation and colony formation assays were performed.

Proliferation assay. The cell growth rate of Hep-G2, Huh-7, Hep-3B and LO2 cells was detected by MTS cell proliferation assay. Cells were seeded in a 96-well plate at a density of $5 \times 10^{2}$ cells/well. The cell growth rate was detected using cell proliferation MTS kit according to the manufacturer's instructions (Promega, USA). Each experiment was performed three times independently.

Colony formation assay. For the colony formation assay, PAQR3-expressing Hep-3B cells or control Hep-3B cells were

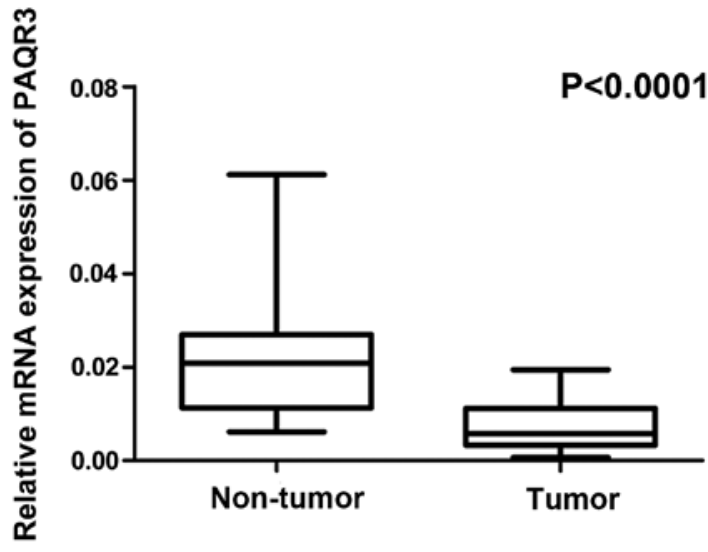

Figure 1. Downregulation of the mRNA expression of PAQR3 in the HCC tissues as assessed by real-time quantitative RT-PCR.

plated in a 6-well plate at a density of $1 \times 10^{3}$ cells/well. After 10 days of culture, surviving colonies ( $>50$ cells/colony) were counted with crystal violet $(0.5 \%)$ staining. Colony-forming efficiency (CFE \%) was defined as the ratio of the number of colonies formed in the culture to the number of cells inoculated. The experiment was conducted three times independently.

Statistical analysis. Statistical analyses were performed using programs including the Statistical Package for the Social Sciences, ver. 17.0 (SPSS Inc, Chicago, IL, USA). Paired-samples t-test was used to compare protein expression of PAQR3 in HCC tissues with paired adjacent non-tumor tissue samples. Comparisons of PAQR3 tumor expression levels with clinicopathological features were evaluated by $\chi^{2}$ tests. Follow-up time was censored if the patient was lost to follow-up. Survival curves were calculated using the Kaplan-Meier method and compared by the log-rank test. Cox proportional-hazard analysis was used for the univariate and multivariate analyses to explore the effect of PAQR3 expression on HCC clinicopathological variables on survival. The two-tailed unpaired Student's t-test was used to assess differences in cell growth rate and colony formation. Differences were considered significant at $\mathrm{P}<0.05$.

\section{Results}

PAQR3 mRNA expression in the primary HCC tissues. Real-time quantitative PCR was conducted on 62 paired surgical samples consisting of HCC (cancerous tissues and the corresponding adjacent non-cancerous tissues from the same patients) to explore expression of PAQR3 at the mRNA level. As shown in Fig. 1, the median level of PAQR3 mRNA expression was significantly lower in the cancer tissues than that in the corresponding adjacent non-tumor tissues $(\mathrm{P}<0.0001)$, and $80.64 \%$ of the subjects (50/62) displayed lower mRNA expression of PAQR3 in cancer tissues. All of the 62 paired tissues were independently tested twice.

$P A Q R 3$ protein expression in the primary HCC tissue samples and cell lines. To investigate whether the expression of PAQR3 was decreased at the protein level, western blotting was performed on the 26 paired HCC cancer tissues and their 
A

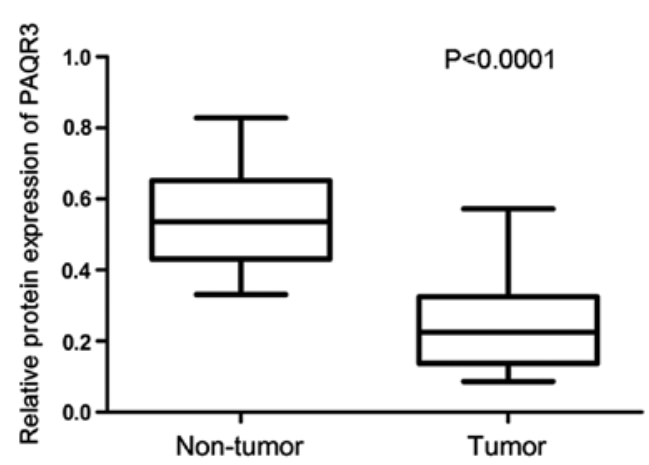

B

Case 1 Case 2 Case 3 Case 4 Case 5 Case 6

$\begin{array}{llllllllllllllllllllll} & \mathrm{T} & \mathrm{N} & \mathrm{T} & \mathrm{N} & \mathrm{T} & \mathrm{N} & \mathrm{T} & \mathrm{N} & \mathrm{T} & \mathrm{N} & \mathrm{T}\end{array}$

PAQR3

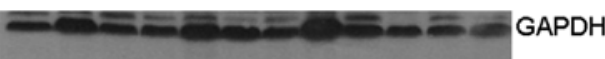

C

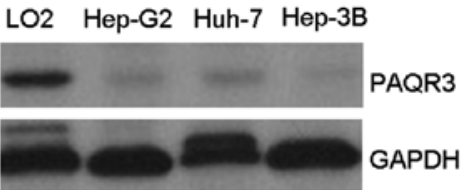

Figure 2. Protein expression of PAQR3 is decreased in the hepatocellular cancer tissues as determined by western blotting. (A) PARQ3 protein expression levels in all of the paired hepatocellular cancer tissues and the matched adjacent non-cancerous tissues from 26 patients. (B) Representative results of the PAQR3 protein expression levels in paired hepatocellular cancer tissues and the matched adjacent non-cancerous tissues from 6 patients. (C) PAQR3 protein expression in hepatic cancer cell lines, Hep-3B, Hep-G2, Huh-7 and normal hepatic cell line LO2.

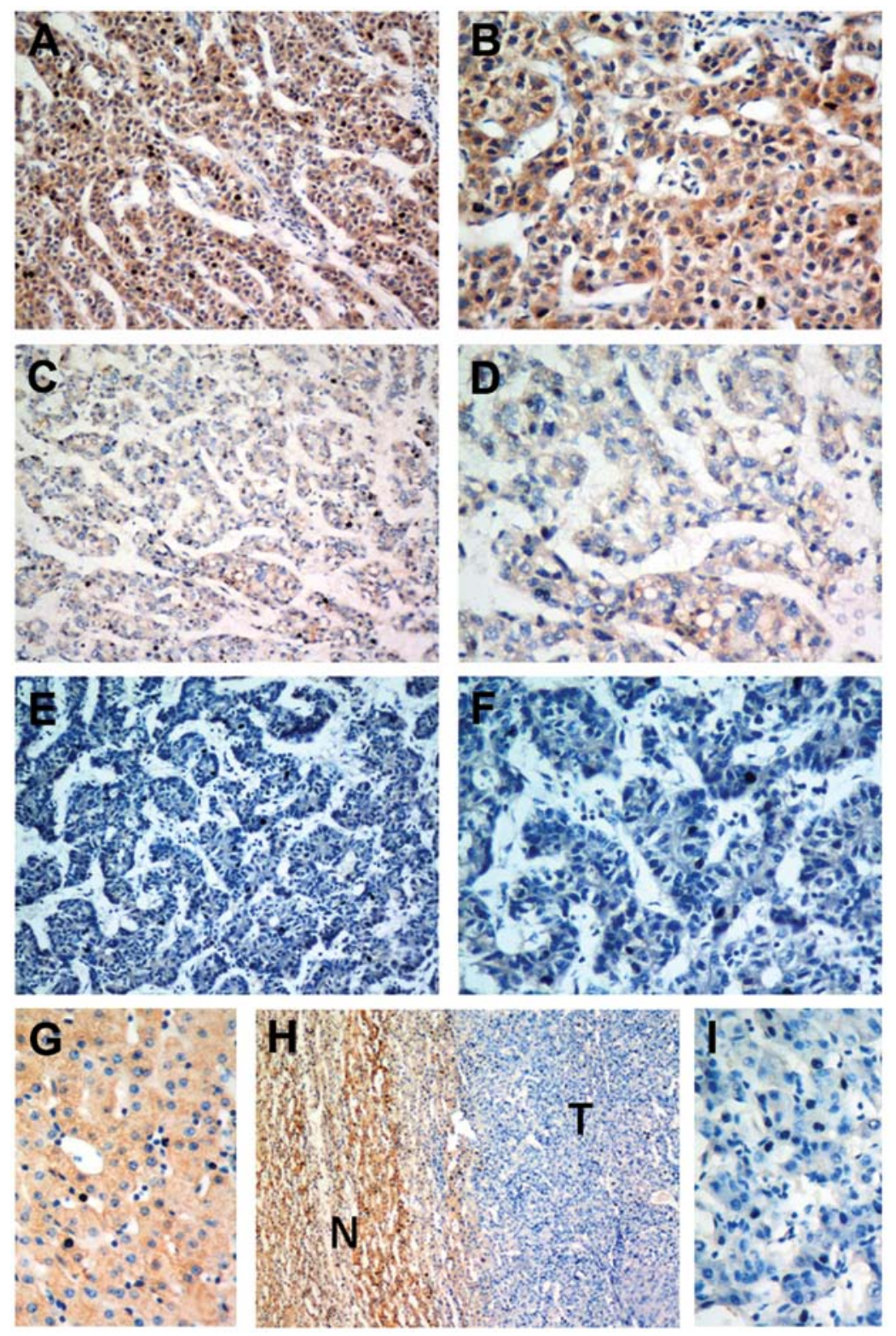

Figure 3. Immunohistochemical analysis of PAQR3 protein expression in primary HCC surgical specimens. (A and B) Well-differentiated HCC scored as PAQR3(++). (C and D) Moderately differentiated HCC scored as PAQR3(++). (E and F) Poorly differentiated HCC scored as PAQR3(-). (H) Normal liver tissue scored as PAQR3(+++). (G-I) Immunostaining of HCC tumor area and the adjacent non-tumor area. N, non-tumor tissue; T, tumor tissue. Magnifications: $\mathrm{H}$, x100; A, C, E, x200; B, D, F, G, I, x400. 
corresponding adjacent non-cancer tissues. Consistent with the qRT-PCR results, western blot analyses showed that PAQR3 expression was decreased in $79.92 \%(20 / 26)$ of cancerous tissues compared with their corresponding non-cancerous tissues $(\mathrm{P}<0.001$, Fig. 2A). As shown in Fig. 2B, 6 HCC cancer tissues exhibited lower PAQR3 expression to some extent, and all of the 6 paired tissues were tested three times independently. Likewise, the PAQR3 protein expression was markedly decreased in the hepatic cancer cell lines, Hep-G2, Huh-7, particularly in Hep-3B, compared with normal hepatic cell line LO2 (Fig. 2C).

PAQR3 expression in HCC and its relationship with clinicopathological features. We further examined PAQR3 expression in a total of 132 HCC surgical specimens using immunohistochemical staining. Among them, 79/132 (59.85\%) cases showed low expression of PAQR3 (PAQR3- or PAQR3+) and 53/132 (40.15\%) cases exhibited high PAQR3 expression (PAQR3++ or PAQR3+++) (Table I). In the positive cases, PAQR3 was detected as being localized in the cytoplasm of the cells (Fig. 3B, D and G). PAQR3 expression was found positive in normal liver tissues (Fig. $3 \mathrm{G}$ and $\mathrm{H}$ ). In cases with adjacent hyperplastic tissue, we often observed a sharp contrast between infiltrative tumor areas of negative staining and the adjacent non-tumor tissue of positive staining (Fig. 3H). Analysis of the relationship between expression of PAQR3 and various clinicopathological parameters is listed in Table I. The expression of PAQR3 was significantly correlated with serum AFP $(\mathrm{P}=0.034)$, mitotic count $(\mathrm{P}=0.012)$, tumor size $(\mathrm{P}=0.028)$, histologic grade $(\mathrm{P}=0.005)$ and recurrence $(\mathrm{P}=0.004)$. Well-differentiated cases showed strongly positive expression (Fig. 3A and B), moderately differentiated cases showed poorly positive expression of PAQR3 (Fig. 3C and D), and in most poorly differentiated cases, we often did not detect PAQR3 expression (Fig. 3E and F). There was no statistically significant difference between PAQR3 expression and age, HBsAg status, liver cirrhosis and metastasis.

Relationship between PAQR3 expression and patient survival. Having demonstrated the correlation of PAQR3 expression with clinicopathological features, we proceeded to examine the relationship between PAQR3 expression and patient survival. The prognostic value of PAQR3 for overall and disease-free survival of HCC patients was evaluated between patients with high and low PAQR3 expression phenotypes. Kaplan-Meier curves showed that, in primary HCC category, patients carrying high PAQR3 expression phenotypes had prolonged overall survival time (77.98 \pm 5.84 months) and disease-free survival (74.58 \pm 6.49 months), whereas patients expressing lower levels of PAQR3 showed a much shorter overall survival time $(53.07 \pm 5.85$ months; log-rank test, $\mathrm{P}=0.009$; Fig. $4 \mathrm{~A})$ and disease-free survival (47.98 \pm 6.37 months; log-rank test, $\mathrm{P}=0.005$; Fig. 4B). The cumulative 5-year overall survival rate was $71.97 \%$ for patients with high levels of PAQR3. Surprisingly, the survival rate dramatically declined to $40.91 \%$ for patients with low levels of PAQR3.

Univariate and multivariate analyses of the prognostic variables in HCC patients. To determine whether PAQR3 could serve as a risk factor with clinical usefulness, univariate and
Table I. Correlation of PAQR3 expression and clinicopathological data in the 132 hapatocarcinoma cases.

\begin{tabular}{|c|c|c|c|c|c|}
\hline \multirow{2}{*}{$\begin{array}{l}\text { Clinicopathological } \\
\text { parameters }\end{array}$} & \multirow[b]{2}{*}{$\mathrm{N}$} & \multicolumn{2}{|c|}{$\begin{array}{c}\text { PAQR3 } \\
\text { expression }\end{array}$} & \multirow[b]{2}{*}{$\chi^{2}$} & \multirow[b]{2}{*}{ P-value } \\
\hline & & Low & High & & \\
\hline All cases & 132 & 79 & 53 & & \\
\hline Age (years) & & & & 0.807 & 0.369 \\
\hline$<50$ & 67 & 42 & 25 & & \\
\hline$\geq 50$ & 65 & 37 & 28 & & \\
\hline HBsAg status & & & & 2.504 & 0.114 \\
\hline Negative & 19 & 10 & 9 & & \\
\hline Positive & 113 & 69 & 44 & & \\
\hline Serum AFP $(\mu \mathrm{g} / \mathrm{l})$ & & & & 4.515 & $0.034^{\mathrm{a}}$ \\
\hline$\leq 400$ & 41 & 19 & 22 & & \\
\hline$>400$ & 91 & 60 & 31 & & \\
\hline Mitotic count & & & & 6.356 & $0.012^{\mathrm{a}}$ \\
\hline$\leq 20$ & 67 & 33 & 34 & & \\
\hline$>20$ & 65 & 46 & 19 & & \\
\hline Liver cirrhosis & & & & 0.556 & 0.456 \\
\hline No & 60 & 38 & 22 & & \\
\hline Yes & 72 & 41 & 31 & & \\
\hline Tumor size $(\mathrm{cm})$ & & & & 4.803 & $0.028^{\mathrm{a}}$ \\
\hline$<5$ & 57 & 28 & 29 & & \\
\hline$\geq 5$ & 75 & 51 & 24 & & \\
\hline Histologic grade & & & & 7.748 & $0.005^{\mathrm{b}}$ \\
\hline Well & 23 & 9 & 14 & & \\
\hline Moderate & 63 & 35 & 28 & & \\
\hline Poor & 46 & 35 & 11 & & \\
\hline Recurrence & & & & 8.100 & $0.004^{\mathrm{b}}$ \\
\hline No & 94 & 49 & 45 & & \\
\hline Yes & 38 & 30 & 8 & & \\
\hline Metastasis & & & & 0.044 & 0.834 \\
\hline No & 111 & 66 & 45 & & \\
\hline Yes & 21 & 13 & 8 & & \\
\hline
\end{tabular}

$\mathrm{N}$, number of samples in each group; ${ }^{\mathrm{a}} \mathrm{P}<0.05$, statistically significant.

multivariate analyses were carried out using Cox proportional hazards model to compare the impact of PAQR3 expression on other clinical pathological features of the HCC patients. Cox regression analyses revealed that a low level of PAQR3 was associated with a significantly increased risk of cancerrelated death $(\mathrm{P}<0.01)$ in HCC patients. The HRs indicated that serum AFP, histological grade and recurrence were unfavorable predictors based on univariate analysis (Tables II and III). After adjusting for potential confounding factors, low expression of PAQR3 in HCC was found to predict poorer survival in an independent manner (Tables II and III). Analyses using multivariate Cox regression model for clinicopathological diagnoses showed that PAQR3 and recurrence 
Table II. Univariate and multivariate analyses of the overall survival in $132 \mathrm{HCC}$ patients.

\begin{tabular}{|c|c|c|c|c|c|c|}
\hline \multirow[b]{2}{*}{ Parameters } & \multicolumn{3}{|c|}{ Univariate analysis } & \multicolumn{3}{|c|}{ Multivariate analysis } \\
\hline & HR & $95 \% \mathrm{CI}$ & P-value & $\mathrm{HR}$ & $95 \% \mathrm{CI}$ & P-value \\
\hline PAQR3 & 0.445 & $0.182-0.793$ & $0.006^{\mathrm{b}}$ & 0.608 & $0.375-0.984$ & $0.010^{\mathrm{a}}$ \\
\hline Age & 0.846 & $0.602-1.103$ & 0.583 & & & \\
\hline HBsAg status & 1.501 & $0.928-2.355$ & 0.206 & & & \\
\hline Serum AFP & 2.271 & $1.329-3.881$ & $0.030^{\mathrm{a}}$ & 1.611 & $0.898-2.890$ & 0.110 \\
\hline Mitotic count & 1.072 & $0.894-2.460$ & 0.225 & & & \\
\hline Liver cirrhosis & 0.812 & $0.654-1.081$ & 0.108 & & & \\
\hline Tumor size & 1.413 & $0.906-2.204$ & 0.127 & & & \\
\hline Histologic grade & 1.840 & $0.223-3.211$ & $0.027^{\mathrm{a}}$ & 0.945 & $0.495-1.808$ & 0.869 \\
\hline Recurrence & 9.173 & $3.052-18.655$ & $<0.001^{\mathrm{c}}$ & 6.492 & $2.153-13.906$ & $<0.001^{\mathrm{c}}$ \\
\hline Metastasis & 1.845 & $0.858-2.638$ & 0.154 & & & \\
\hline
\end{tabular}

HR, hazard ratio; $\mathrm{CI}$, confidence interval; ${ }^{\mathrm{P}}<0.05$, statistically significant.

Table III. Univariate and multivariate analyses of the disease-free survival in $132 \mathrm{HCC}$ patients.

\begin{tabular}{|c|c|c|c|c|c|c|}
\hline \multirow[b]{2}{*}{ Parameters } & \multicolumn{3}{|c|}{ Univariate analysis } & \multicolumn{3}{|c|}{ Multivariate analysis } \\
\hline & HR & $95 \% \mathrm{CI}$ & P-value & HR & $95 \% \mathrm{CI}$ & $\mathrm{P}$-value \\
\hline PAQR3 & 0.395 & $0.155-0.623$ & $0.004^{\mathrm{b}}$ & 0.425 & $0.307-0.722$ & $0.006^{\mathrm{b}}$ \\
\hline Age & 0.805 & $0.594-1.235$ & 0.571 & & & \\
\hline HBsAg status & 1.959 & $0.779-4.981$ & 0.158 & & & \\
\hline Serum AFP & 3.199 & $1.624-4.302$ & $0.021^{\mathrm{a}}$ & 1.416 & $0.539-2.312$ & 0.267 \\
\hline Mitotic count & 1.035 & $0.923-2.211$ & 0.232 & & & \\
\hline Liver cirrhosis & 0.922 & $0.637-1.334$ & 0.136 & & & \\
\hline Tumor size & 1.381 & $0.308-4.316$ & 0.096 & & & \\
\hline Histologic grade & 1.552 & $0.969-2.986$ & $0.043^{\mathrm{a}}$ & 1.032 & $0.532-2.485$ & 0.553 \\
\hline Recurrence & 7.786 & $4.052-15.304$ & $<0.001^{\mathrm{c}}$ & 6.655 & $3.863-10.574$ & $<0.001^{\mathrm{c}}$ \\
\hline Metastasis & 2.035 & $1.326-3.085$ & 0.095 & & & \\
\hline
\end{tabular}

HR, hazard ratio; CI, confidence interval; ${ }^{\mathrm{a}} \mathrm{P}<0.05$, statistically significant.

were independent prognostic parameters and predicted poor overall survival and disease-free survival. Thus, PAQR3 expression may play a role in predicting overall survival and disease-free survival of HCC patients (Tables II and III).

Role of PAQR3 in cell proliferation and colony formation in the LO2 and Hep-3B cell lines. To evaluate the effects of PAQR3 on cell proliferation, the PAQR3 expression vector and the control vector were respectively transfected into Hep-3B cells. PAQR3 expression in the transfected cells was detected by western blotting (Fig. 5A). The cell growth assay revealed that the cell growth rate of the PAQR3-transfected Hep-3B cells was significantly lower than that of the control vectortransfected Hep-3B cells (Fig. 5D). Meanwhile, the efficiency of colony formation was significantly $(\mathrm{P}=0.0379)$ inhibited in the PAQR3-transfected hepatic cancer cells compared with control vector-transfected hepatic cancer cells (Fig. 5C).
To further confirm the proliferation suppression function of PAQR3, we silenced PAQR3 expression in the LO2 cell line with siRNA. PAQR3 expression in the transfected cells was detected by western blotting (Fig. 5B). We found that silencing of the expression of PAQR3 in LO2 cells significantly enhanced cell proliferation when compared with rates of cell proliferation in the mock siRNA-treated cells (Fig. 5E).

\section{Discussion}

PAQR3 has been identified as a tumor-suppressor gene in many cancer cell lines in vitro, as overexpression of PAQR3 results in the inhibition of cell proliferation, migration, sprouting and angiogenesis, and knockdown of PAQR3 promotes cell proliferation, migration, sprouting and angiogenesis $(11,12,14)$. In a study by Xie et al, the number and size of papillomas were increased in PAQR $3^{-/-}$mice, accompanied by shortened tumor 
A

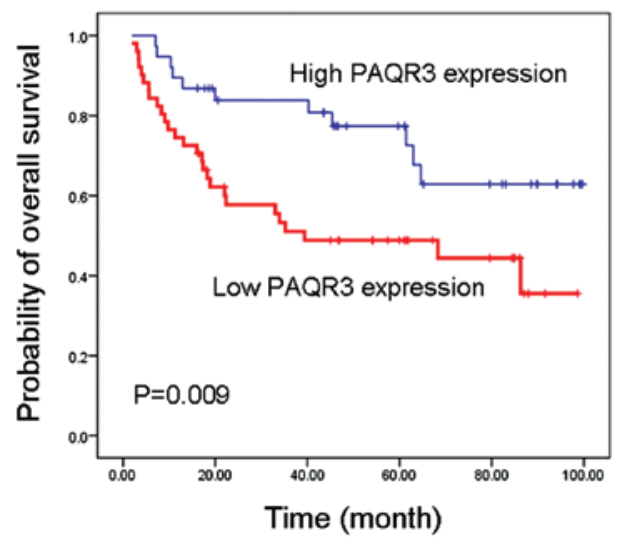

$\mathrm{B}$

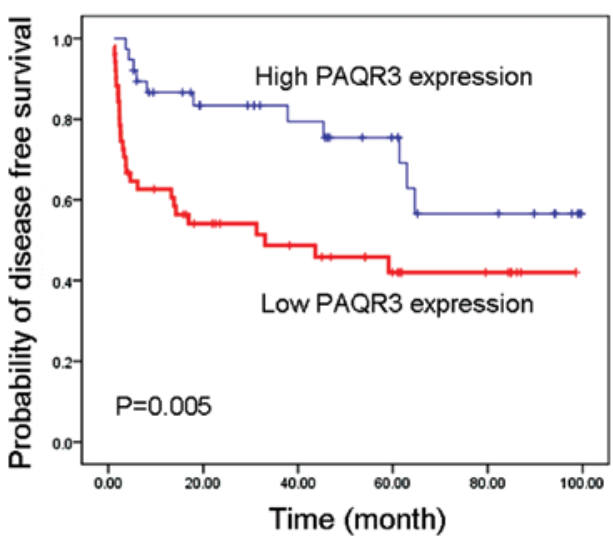

Figure 4. Kaplan-Meier survival analysis of primary HCC patients ( $=132)$ after surgical resection with low PAQR3 expression (n=76) and high PAQR3 expression ( $\mathrm{n}=56$ ). (A) The overall survival of patients in the PAQR3 low expression group was significantly lower than the overall survival of patients in the PAQR3 high expression group. (B) Patients with PAQR3 low expression showed shorter disease-free survival than PAQR3 high expression patients.

A

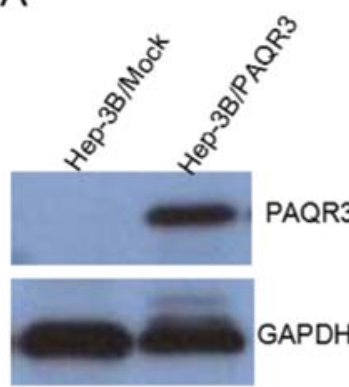

B

$\mathrm{C}$

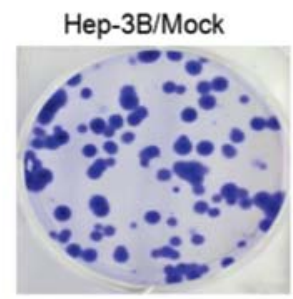

Hep-3B/PAQR3
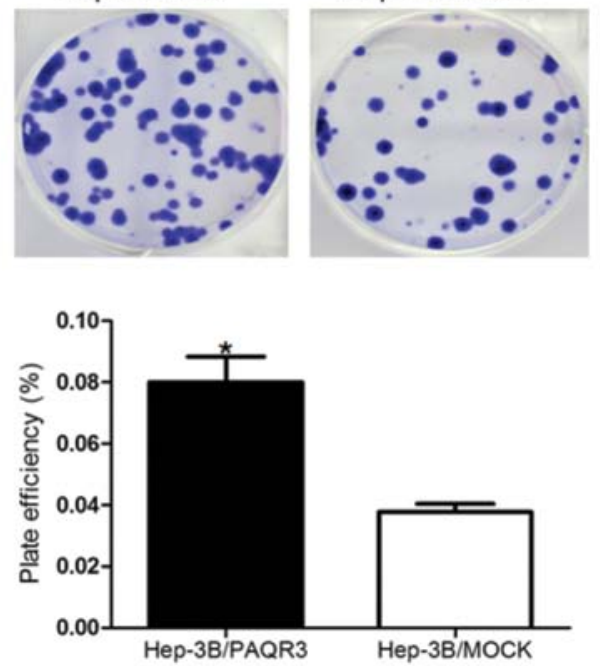

D

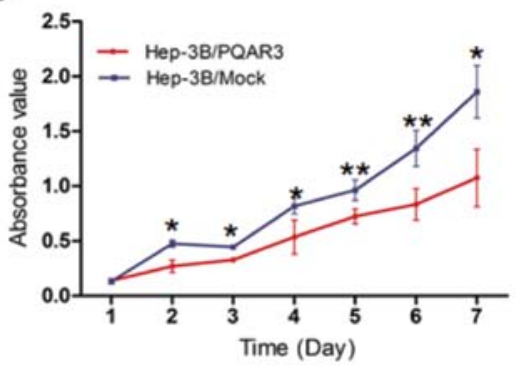

E

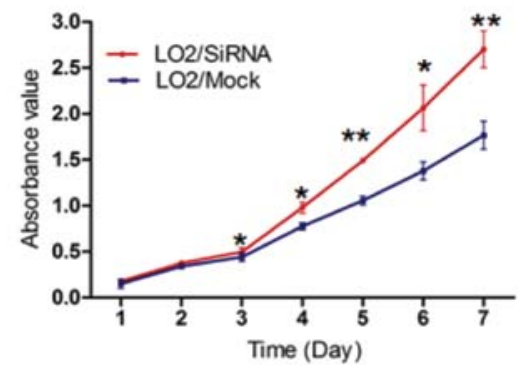

Figure 5. The growth-suppressor role of PAQR3 in cell proliferation and colony formation assays of LO2 and Hep-3B cell lines. (A) Western blot analysis of PAQR3 expression in Hep-3B cells transfected with the PAQR3 plasmid (Hep-3B/PAQR3). (B) Western blot analysis of PAQR3 expression in LO2 cells with silenced PAQR3 expression (LO2/SiRNA). (D) Cell proliferation assay showing the suppressive effect of restoring PAQR3 expression on the proliferation of the Hep-3B cell line. (C) PAQR3 inhibited the colony formation of Hep-3B/PAQR/3 cells. Images are shown (upper panels), and quantitative analyses of plaque numbers are shown as the mean values $\pm \mathrm{SD}$ (lower histogram). (E) Cell proliferation assay showing significantly enhanced proliferation rate of PAQR3silenced LO2/SiRNA cells compared with mock siRNA treated LO2 cells (LO2/mock). ${ }^{*} \mathrm{P}<0.05,{ }^{* *} \mathrm{P}<0.01$.

latency and enhanced keratinocyte proliferation (12). The possible mechanism is associated with its regulation of the ERK signaling pathway, and suppressing mitogen-activated protein kinase signaling, negatively regulates the transactivation activity of hypoxia-inducible factor $1 \alpha(\mathrm{HIF}-1 \alpha)$ by inhibiting formation of the HIF-1 $\alpha /$ p300 complex and suppressing VEGF transcription, thereby reducing hypoxia-induced VEGF production $(11,12,14)$. Our observations, presented here for the 
first time, indicate that PAQR3 acts as a tumor-suppressor gene in human HCC.

In the present study, we examined the expression of PAQR3 at the mRNA and protein levels in paired primary HCC samples using qRT-PCR and western blotting. The results demonstrated that $\mathrm{PAQR} 3$ was downregulated in the majority of the primary HCC cancer tissues at both the transcriptional and translational levels. Consistently, immunohistochemical analyses also showed that PAQR3 expression was decreased in most HCC cancer tissues compared with their corresponding non-cancer tissues. We favor the hypothesis that a serious alteration, insertion and/or deletion in the gene may possibly result in diminished or abolished expression of PAQR3 in HCC, which will require further investigation in future research. These results indicated that downregulated expression of PAQR3 may play a role in $\mathrm{HCC}$ carcinogenesis.

In the immunohistochemical analysis, downregulation of PAQR3 expression in HCC was significantly associated with AFP, mitotic count, tumor size, histologic grade and recurrence. Mitotic count is used in HCC to quantify proliferation rates, and low PAQR3 expression was related with a high mitotic count. Fan et al demonstrated that knockdown of PAQR3 enhanced malignant melanoma cell proliferation and motility via Ras/Raf/MEK/ERK signaling cascade phosphorylation (7). The relationship between low PAQR3 expression and large tumor size in the present study suggested that the lack of PAQR3 may facilitate rapid expansion of the tumor. Additionally, most well-differentiated HCC tissues showed high PAQR3 expression, in contrast with a profoundly lower PAQR3 expression in the moderately and poorly differentiated tumor samples. Recently, Jiang et al demonstrated that there exists a functional interplay between PAQR 3 and p53 in EMT and tumorigenesis in HepG2 epithelial cells (11). These findings indicate that loss of PAQR3 expression may suppress the differentiation of HCC cells and further promote the recurrence of HCC. The low expression of PAQR3 was associated with a high serum AFP level, which, to the best of our knowledge, has not been reported, yet the exact mechanism still remains unclear, thus requiring further investigation. Wang et al found PAQR3 downregulation in colorectal cancers, resulting in increased tumor grade in vivo (10). These observations are consistent with our clinicopathological findings that low expression of PAQR3 in HCC is associated with larger tumor size. Our findings, together with the findings of other authors, strongly suggest that PAQR3 may be a novel, yet important, prognostic marker for HCC patients.

Kaplan-Meier survival analysis revealed that low PAQR3 expression was significantly correlated with a poor prognosis of HCC patients after surgical resection. Furthermore, PAQR3 expression was found to be an independent prognostic factor relative to overall and disease-free survival using multivariate analysis. Consistent with our observations, downregulation of PAQR3 expression in colorectal cancer was found to be significantly associated with a poor survival status of colorectal cancer patients (10). These findings suggest that PAQR 3 may serve as a new and independent predictor of prognosis for HCC patients after surgical resection.
We further investigated the functional role of PAQR3 in hepatic cell lines. Restoring PAQR3 expression in Hep-3B cells significantly inhibited cell proliferation and colony formation. Silencing the expression of PAQR3 in LO2 cells significantly enhanced the cell growth rate. These results indicated that PAQR3 may play an important role in inhibiting tumor cell growth. Recently, functional assays of PAQR3 in colorectal cancer cell lines by Wang et al suggest that PAQR3 exerts tumor-suppressor activity (10). Fan et al demonstrated that restoring the expression of wild-type PAQR3 inhibits ERK activation, cell proliferation and transformation of A375 cells in vitro, and is sufficient to suppress tumorigenicity of xenografts with human malignant melanoma cell line A375 harboring B-Raf mutation V600E (7), while RNA interferencemediated PAQR3 silencing enhances cellular proliferation migration, sprouting and angiogenesis of endothelial cells (12). Notably, knockdown of PAQR3 led to enhancement of LPA-stimulated AKT and GSK3 $\beta$ phosphorylation, together with increased accumulation of $\beta$-catenin and the appearance of EMT features that were antagonized by p53 overexpression in HepG2 epithelial cells (15). Aberrant mTOR signaling has been detected in up to $48 \%$ of HCCs and represents a suitable therapeutic target in HCC (16), indicating a link between PAQR3 and the AKT/MTOR signaling pathway in HCC carcinogenesis and further investigation is warranted. These data, together with ours, suggest that loss of PAQR3 may play an important role in the process of carcinogenesis.

Collectively, our results demonstrate that PAQR3 expression is downregulated in the majority of HCC tissues at both the mRNA and protein levels, and low PAQR3 expression may indicate a dismal prognosis of HCC patients. In addition, we confirmed that PAQR3 inhibits tumor cell growth and colony formation in vitro. To date, the data generated in the present study represent the first findings to correlate the presence of PAQR3 with clinicopathological characteristics and the survival of HCC patients. Our findings provide evidence that PAQR3 may be a novel prognostic biomarker for HCC and may serve as a potential therapeutic candidate for $\mathrm{HCC}$.

\section{Acknowledgements}

This study was supported by the Union Hospital Key Laboratory Foundation of Biological Target Therapy (no. 02.03.2013-80 to P.D.L.) and the Independent Innovation Research Foundation of Huazhong University of Science and Technology (no. 01-08530059 to P.D.L.).

\section{References}

1. Parkin DM, Bray F, Ferlay J, et al: Estimating the world cancer burden: Globocan 2000. Int J Cancer 94: 153-156, 2001.

2. He J, Gu D, Wu X, et al: Major causes of death among men and women in China. N Engl J Med 353: 1124-1134, 2005.

3. Thomas MB and Zhu AX: Hepatocellular carcinoma: The need for progress. J Clin Oncol 23: 2892-2899, 2005.

4. Zender L, Villanueva A, Tovar V, et al: Cancer gene discovery in hepatocellular carcinoma. J Hepatol 52: 921-929, 2010.

5. Feng L, Xie X, Ding Q, et al: Spatial regulation of Raf kinase signaling by RKTG. Proc Natl Acad Sci USA 104: 14348-14353, 2007.

6. Luo X, Feng L, Jiang X, et al: Characterization of the topology and functional domains of RKTG. Biochem J 414: 399-406, 2008. 
7. Fan F, Feng L, He J, et al: RKTG sequesters B-Raf to the Golgi apparatus and inhibits the proliferation and tumorigenicity of human malignant melanoma cells. Carcinogenesis 29: 1157-1163, 2008.

8. Zhang Y, Jiang X, Qin X, et al: RKTG inhibits angiogenesis by suppressing MAPK-mediated autocrine VEGF signaling and is downregulated in clear-cell renal cell carcinoma. Oncogene 29: 5404-5415, 2010.

9. Jiang Y, Xie X, Zhang Y, et al: Regulation of G-Protein signaling by RKTG via sequestration of the $\mathrm{G}$ betagamma subunit to the Golgi apparatus. Mol Cell Biol 30: 78-90, 2010.

10. Wang X, Li X, Fan F, et al: PAQR3 plays a suppressive role in the tumorigenesis of colorectal cancers. Carcinogenesis 33 : 2228-2235, 2012.

11. Jiang Y, Xie X, Li Z, et al: Functional cooperation of RKTG with p53 in tumorigenesis and epithelial-mesenchymal transition. Cancer Res 71: 2959-2968, 2011.
12. Xie X, Zhang Y, Jiang Y, et al: Suppressive function of RKTG on chemical carcinogen-induced skin carcinogenesis in mouse. Carcinogenesis 29: 1632-1638, 2008.

13. Grabinski N, Ewald F, Hofmann BT, et al: Combined targeting of AKT and mTOR synergistically inhibits proliferation of hepatocellular carcinoma cells. Mol Cancer 20: 11, 2012.

14. Psyrri A, Arkadopoulos N, Vassilakopoulou M, et al: Pathways and targets in hepatocellular carcinoma. Expert Rev Anticancer Ther 12: 1347-1357, 2012.

15. Jain S, Singhal S, Lee P, et al: Molecular genetics of hepatocellular neoplasia. Am J Transl Res 2: 105-118, 2010.

16. Guichard C, Amaddeo G, Imbeaud S, et al: Integrated analysis of somatic mutations and focal copy-number changes identifies key genes and pathways in hepatocellular carcinoma. Nat Genet 44: 694-698, 2012. 